\title{
Cervical Nontuberculous Mycobacterial Lymphadenitis Mimicking a Thyroid Tumor and Infiltrating Deep into the Neck ${ }^{*}$
}

\author{
Kazuhiro Mino ${ }^{1}$, Tadao Okada $^{1}$, Shouhei Honda ${ }^{1}$, Hisayuki Miyagi ${ }^{1}$, Nobuhisa Ishiguro ${ }^{2}$, \\ Kanako C. Kubota ${ }^{3}$, Taketomi Akinobu ${ }^{1}$ \\ ${ }^{1}$ Department of Gastroenterological Surgery I, Hokkaido University Graduate School of Medicine, Sapporo, Japan \\ ${ }^{2}$ Department of Pediatrics, Hokkaido University Graduate School of Medicine, Sapporo, Japan \\ ${ }^{3}$ Department of Surgical Pathology, Hokkaido University Hospital, Sapporo, Japan \\ Email: okadata@med.hokudai.ac.jp
}

Received August 14, 2012; revised September 16, 2012; accepted September 27, 2012

\begin{abstract}
Aim: Nontuberculous Mycobacterial Lymphadenitis (NML), which occurs in 1.2 per 100,000 children, is very rare. And those which emerge at the anterior cervical portion and infiltrate deep into the neck are even more rare. Generally, this disorder is uncommon existed near the thyroid gland. We report here a case of NML mimicking a thyroid tumor and infiltrating into the deep part of the anterior neck. Case: A mass at the anterior portion of her neck was found at 5 years old. It was not mobile and palpated as an irregularly surfaced hard mass whose size was $3 \mathrm{~cm}$ at the anterior lower portion of her neck. Ultrasonography showed an oval mass which existed near the slightly inferior part of the right lobe of the thyroid gland. Enhanced computed tomography showed a mass near the slightly inferior part of the right lobe of the thyroid gland. The mass was resected with the platysma and the right sternohyoid muscle. In the HE staining, epithelioid cell and Langhans type giant cells surrounding coagulative necrosis lesions which seemed to be caseation necrosis existed, similarly to cervical NML. Discussion: No consensus exists for the treatment of NML, but many documents advise complete excision. When the lesion cannot be completely removed, excision as far as possible and additional antibiotics are recommended. The characteristics of imaging of NML around the thyroid gland and infiltrating deep into the anterior neck and mediastinum are discussed.
\end{abstract}

Keywords: Anterior Cervical Portion; Mediastinum; Nontuberculous Mycobacterial lymphadenitis; Thyroid Gland

\section{Introduction}

Nontuberculous Mycobacterial Lymphadenitis (NML) is a very rare and an uncommon cause of cervical lymphadenitis in children. And those which emerge at the anterior cervical portion and infiltrate deep into the neck are even more rare. Although complete surgical excision of the affected region has been the accepted therapy based on its superior cure rate, recent reports have suggested that medical therapy may have a role in the treatment of this disease [1]. Generally, this disorder is uncommon existed near the thyroid gland. We report here a case of a 5-year-old girl with NML that emerged around the thyroid gland and infiltrated deep into the neck and mediastinum. We review the literature of this type of disorder and discuss the characteristics of imaging and the problems associated with the surgical and medical treatments.

*Corresponding author received no funding for this report and declare no conflict of interest.

\section{Case Report}

A mass at the anterior portion of her neck was found by her mother at 5 years old. On admission, it was not mobile and palpated as an irregularly surfaced hard mass whose size was $3 \mathrm{~cm}$ at the anterior lower portion of her neck. Her blood chemistry values were within normal limits. The Mantoux test was done and was slightly positive. Plain chest X-ray showed no abnormal findings. Ultrasonography showed an oval mass which existed near the slightly inferior part of the right lobe of the thyroid gland. Enhanced Computed Tomography (CT) showed a mass near the slightly inferior part of the right lobe of the thyroid gland (Figure 1). The content of the mass was low density, and the mass contained a dissepiment configuration and had a mural enhancement effect on the enhanced CT. The border of this lesion was unclear and the mass was $3.2 \times 2.5 \times 2.6 \mathrm{~cm}$ in diameter. The mass extended from the inferior pole of the right lobe of the thyroid gland to the dorsum of the upper margin of the 


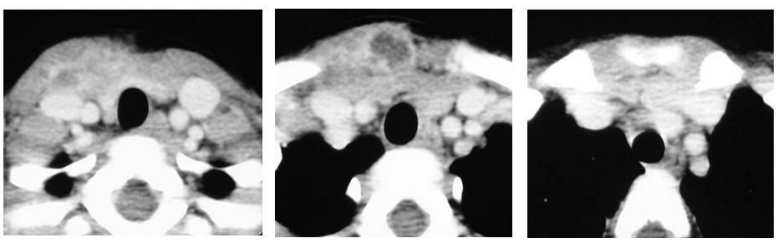

Figure 1. Enhanced Computed Tomography (CT) showed a mass at the slightly inferior portion of the right lobe of thyroid gland. The content of the mass was low $C T$ value and the mass contained dissepiment configuration and had a mural enhancement effect. The mass extended from the inferior pole of the right lobe of the thyroid gland to the dorsum of the upper margin of the sternum.

sternum.

She was operated on with a diagnosis of unknown cervi cal tumor. The tumor invaded the platysma and the right sternohyoid muscle. We therefore resected the mass with those muscles (Figure 2). The intraoperative frozen section histopathological diagnosis was acid fast bacillus infection, based on the findings of hyperplasia of the epithelioid cell which seemed to surround caseation necrosis with the Langhans-type giant cell. In the HE staining, epithelioid cell and Langhans type giant cells surrounding coagulative necrosis lesions which seemed to be caseation necrosis existed, similarly (Figure 2 ). We could could recognize a few bacteria in Oraminrodamin staining.

The results of the general bacterial culture of the resected tumor and postoperative drain discharge were negative, but the Ziehl Neelsen stain of the resected tumor was positive, Gaffky 1. For sputum, gastric juice and drain discharge, bacterial cultures were negative. According to the polymerase chain reaction, the results of Mycobacterium tuberculosis, Mycobacterium Avium and Mycobacterium intracellurae were negative in the resected mass tissue, sputum, gastric juice, and drain discharge. Based on these findings, the final diagnosis was cervical NML.

After discharge, she had been prescribed clarithromycin $15 \mathrm{mg} / \mathrm{kg} /$ day for 2 months. She dropped out of our follow-up because of familial issues.

\section{Discussion}

NTM lymphadenitis is very rare, and occurs in from 1.2 to 77 children per 100,000 [2,3]. However, this disease has shown an increasing tendency recently, and sometimes shows development to the cervical deep part and mediastinal space such as in our case. A report shows $61 \%$ submandibular, $11.8 \%$ preauricular, $11 \%$ anterior cervical, $2.5 \%$ posterior triangle, 5.9\% laryngopharyngeal, $2.5 \%$ axillary, $1.6 \%$ inguinal, $0.9 \%$ submental, $0.9 \%$ buccal, $0.9 \%$ subclavian, $0.9 \%$ thigh [4]. Therefore, cases of the anterior region of the neck such as our case are rare.

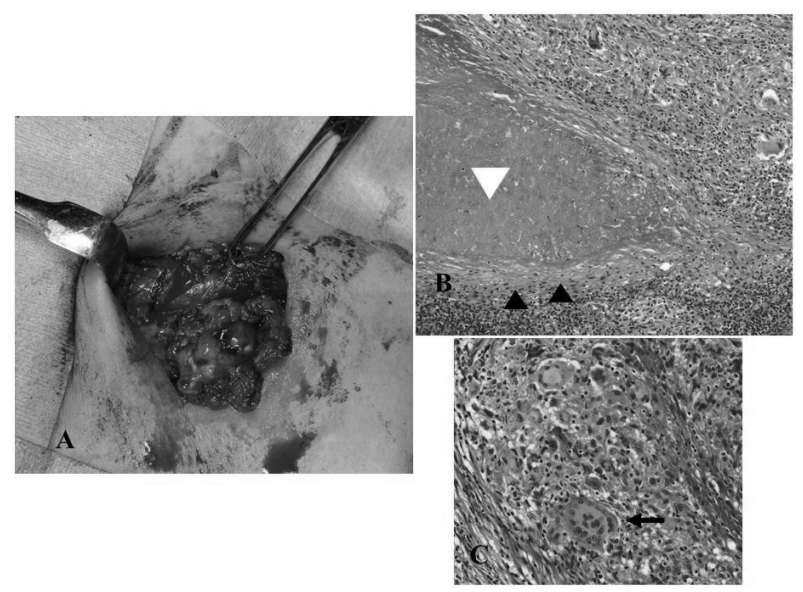

Figures 2. (A) Operation picture of the neck tumor. The tumor invaded the platysma and the right sternohyoid muscle, so we resected the mass with those muscles; (B), (C) The pathology of $\mathrm{HE}$ staining showed epithelioid cells (black arrow head) and Langhans type giant cells (black arrow) surrounding a coagulative necrotic lesion which seems to be caseation necrosis (white arrow head).

As to the treatment of mycobacterial infection, the first line is surgical intervention. The cure ratio is reported tobe $74 \%$-more than $99 \%$ by complete excision, $33 \%$ $92 \%$ by curettage [4], and $0 \%-27 \%$ by incision and drainage [5], so it is thought that we require excision or at least curettage. However, when there is a risk for damage of a critical portion or for the tumor to reach the cervical deep part such as in our case, we need to resect the mass partially and require antibiotic therapy. The rate of re-operation after administration of postoperative antibiotics varies widely from $0 \%$ to $42 \%$. There is no consensus regarding the postoperative use of antibiotics.

\section{Conclusion}

In conclusion, we experienced a very rare case of NML which emerged around the thyroid gland and infiltrated into the deep part of the neck and mediastinum mimicking a thyroid tumor. We should perform resection as far as possible, and perform additional antibiotic therapy.

\section{REFERENCES}

[1] J. B. Coulter, D. A. Lloyd, M. Jones, J. C. Cooper, M. S. McCormick, R. W. Clarke and M. I. Tawil, "Nontuberculous Mycobacterial Adenitis: Effectiveness of Chemotherapy Following Incomplete Excision,” Act Pediatrica, Vol. 95, No. 2, 2006, pp.182-188. doi:10.1080/08035250500331056

[2] D. Sigalet, G. Lees and A. Fanning, “Atypical Tuberculosis in the Pediatric Patient: Implication for the Pediatric Surgeon,” Journal of Pediatric Surgery, Vol. 27, No. 11, 1992, pp. 1381-1384. doi:10.1016/0022-3468(92)90181-6

[3] J. A. Lindeboom, E. J. Kuijper, E. S. B. van Coppenraet, R. 
Lindeboom and J. M. Prins, "Surgical Excision versus Antibiotic Treatment for Nontuberculous Mycobacterial Cervicofacial Lymphadenitis in Children: A Multicenter, Randomized, Controlled Trial," Clinical Infectious Diseases, Vol. 44, No. 8, 2007, pp. 1057-1064. doi:10.1086/512675

[4] I. Mushtaq and H. Martin, "Atypical Mycobacterial Disease in Children: A Personal Series,” Pediatric Surgery
International, Vol. 18, No. 8, 2002, pp. 707-711.

[5] B. Elizabeth and K. Y. Murray, "Management of Nontuberculous Mycobacterial Cervical Adenitis,” The Pediatric Infectious Disease Journal, Vol. 19, No. 7, 2000, pp. 674-675. 